\title{
MINI-REVIEW
}

\section{The Role of the Fibroblast in Inflammatory Upper Airway Conditions}

\author{
Stephen L. Ball, ${ }^{*}{ }^{\dagger}$ Derek A. Mann, ${ }^{*}$ Janet A. Wilson, ${ }^{\dagger}$ and Andrew J. Fisher*
}

From the Fibrosis Research Group, * Institute of Cellular Medicine, Newcastle University, Newcastle upon Tyne; and the Department of Otolaryngology Head and Neck Surgery, ${ }^{\dagger}$ Freeman Hospital, Newcastle upon Tyne, United Kingdom

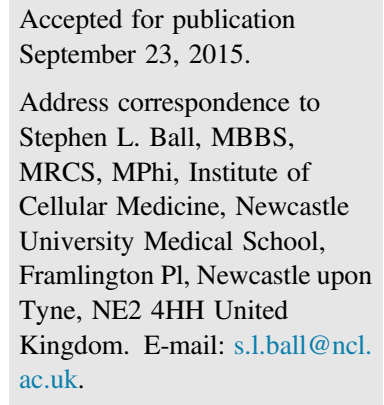

\begin{abstract}
Upper airway inflammation is one of the most frequent health care presentations. This is perhaps not surprising with our exposure to a myriad of environmental microbes, pollutants, and allergens. The precise pathophysiological mechanisms that cause persistent, exaggerated, upper airway inflammation rather than acute resolving illness remain unclear. Analysis of upper airway specimens identifies specific inflammatory cells, cytokine signatures, and fibrotic airway remodeling. Recent research has highlighted the role of stromal cells in the generation and persistence of chronic inflammation. Rather than simply being scaffolding or extracellular matrix-secreting cells on which organ systems are built, stromal cells including fibroblasts and osteocytes have their own independent immunologic functions. Here, we review the emerging inflammatory roles of upper airway fibroblasts, the majority of which appear to influence immune cell chemotaxis and amplify the inflammatory response. (Am J Pathol 2016, 186: 225-233; http://dx.doi.org/10.1016/j.ajpath.2015.09.020)
\end{abstract}

Inflammatory conditions of the upper airways represent some of our most common reasons for seeking health care. Often mistakenly, they are considered trivial conditions yet have significant morbidity, impact on quality of life, and medical expenditure. The precise mechanisms underpinning upper airway inflammation are the subject of considerable investigation. After the emergence of fibroblasts as a source of diverse tissue inflammation, ${ }^{1-3}$ we propose that upper airway fibroblasts drive and significantly contribute to the inflammatory phenotype in the upper airways.

A number of organs show fibroblast-driven inflammatory processes $^{2}$ with typical profibrotic and inflammatory diseases, including but not limited to lung, liver, bowel, joint, and kidney fibrosis. Examples of core processes of fibrotic disease are the remodeling of matrix toward a fibrillarcollagen-rich matrix, first involving production of collagen I/III by myofibroblasts followed by blockade of collagen degradation by fibroblast-derived tissue inhibitor of matrix metalloproteinases 1 , which acts as a specific inhibitor of collagenases. In addition, the matrix enzyme lysyl oxidase like-2 can promote maturation of fibrotic matrix in the maintenance of pathologically inflamed fibroblasts and matrix stiffening through the cross-linking of collagen fibrils as seen in mouse models of lung, liver, and subcutaneous tissue. ${ }^{4}$ In the musculoskeletal system it is proposed that fibroblasts define the cellular and molecular basis for organspecific chronic inflammation. ${ }^{3}$ In the kidney, stromal cells such as fibroblasts and fibrocytes have been shown to contribute to inflammation by enhancing leukocyte infiltration, and contribute to activation by releasing proinflammatory cytokines and via epigenetic modifications such as hypermethylation. ${ }^{1}$

Because of the organization of health care services, human disease research often is geared around the study of a particular organ. If the core mechanisms of fibrosis and inflammation are understood and applicable in anatomically different tissue sites it would allow aspects of fibrosis research to be performed where access to disease tissue is simplest. With the exception of the skin, the sinonasal mucosa is among the most accessible epithelial surface in the body. Access to material for cell, tissue, and microbiological research can be provided by a variety of brushings,

Supported by Wellcome Trust Clinical Research Training Fellowship grant 100201/Z/12/A (S.L.B.).

Disclosures: None declared. 
small biopsies, mucosal scrapes, or swabs from the clinic. The nose thus offers a convenient portal for the study of generic inflammatory mechanisms and the introduction of novel therapies. Sinonasal disease surveillance is likewise very straightforward because office-based endoscopic nasal assessment readily supplements well-validated disease symptom scores. Sinonasal medications often are applied topically via a nasal aerosol, with minimal systemic exposure.

Within the respiratory system, idiopathic pulmonary fibrosis represents the most common progressive interstitial lung disease, characterized by proliferation of fibroblasts and deposition of extracellular matrix. Once established, the disease typically progresses to respiratory failure. The postulated pathogenesis is of repetitive respiratory epithelial injury, followed by airway remodeling and an increase in mesenchymal cells including fibroblasts and activated myofibroblasts, either by proliferation of pre-existing resident cells or via epithelial to mesenchymal transition. ${ }^{5}$ Accumulation of fibroblasts is associated with an increase in chronic inflammation. Similar epithelial cell loss, fibroblast accumulation, and chronic inflammation are seen in upper airway inflammatory diseases. There are no in vivo idiopathic pulmonary fibrosis models that mirror human disease progression, and animal models of upper airway inflammation are similarly limited. Human studies targeting the fibroproliferation in idiopathic pulmonary fibrosis have shown initial promise, although no superiority to placebo in randomized controlled trials of prednisolone, azathioprine, interferon- $\gamma$, anti-tumor necrosis factor (TNF)- $\alpha$, and endothelin-receptor antagonists has been shown. Focus now is shifting toward specific fibroblast-targeted therapies. Lower airway tissue samples can be isolated using a variety of methods such as bronchoscopic-guided biopsy or cellular brushings, although in patients with decreasing respiratory function these are not without risk. However, because the upper airways are lined by the same respiratory epithelium, it would be possible to use the more accessible sinonasal epithelium as a window into the mechanisms of disease further down the airway.

The nose is the first point of contact between environmental microbes, pollutants, and allergens and the respiratory system, and therefore it is not surprising that upper airway inflammation is so common (Figure 1). Chronic rhinosinusitis (CRS) is the most common upper airway illness presentation. Epidemiologic data indicate that the prevalence of CRS in the general population is approximately $12 \%,{ }^{6,7}$ with annual direct health care costs in excess of $\$ 5.8$ billion $^{8}$ in the United States. The high cost of treatment reflects the vast number of affected individuals, chronicity of symptoms, and the percentage of patients who are refractory to current maximal medical management ${ }^{9}$ and require surgical intervention. ${ }^{10}$ Current maximal medical therapy comprises corticosteroids, either intranasal, systemic, or both, in combination with antibiotics and nasal irrigation. ${ }^{11}$ Failure of medical therapy typically results in patients having functional endoscopic sinus surgery to resect diseased tissue, ventilate affected sinus groups, and

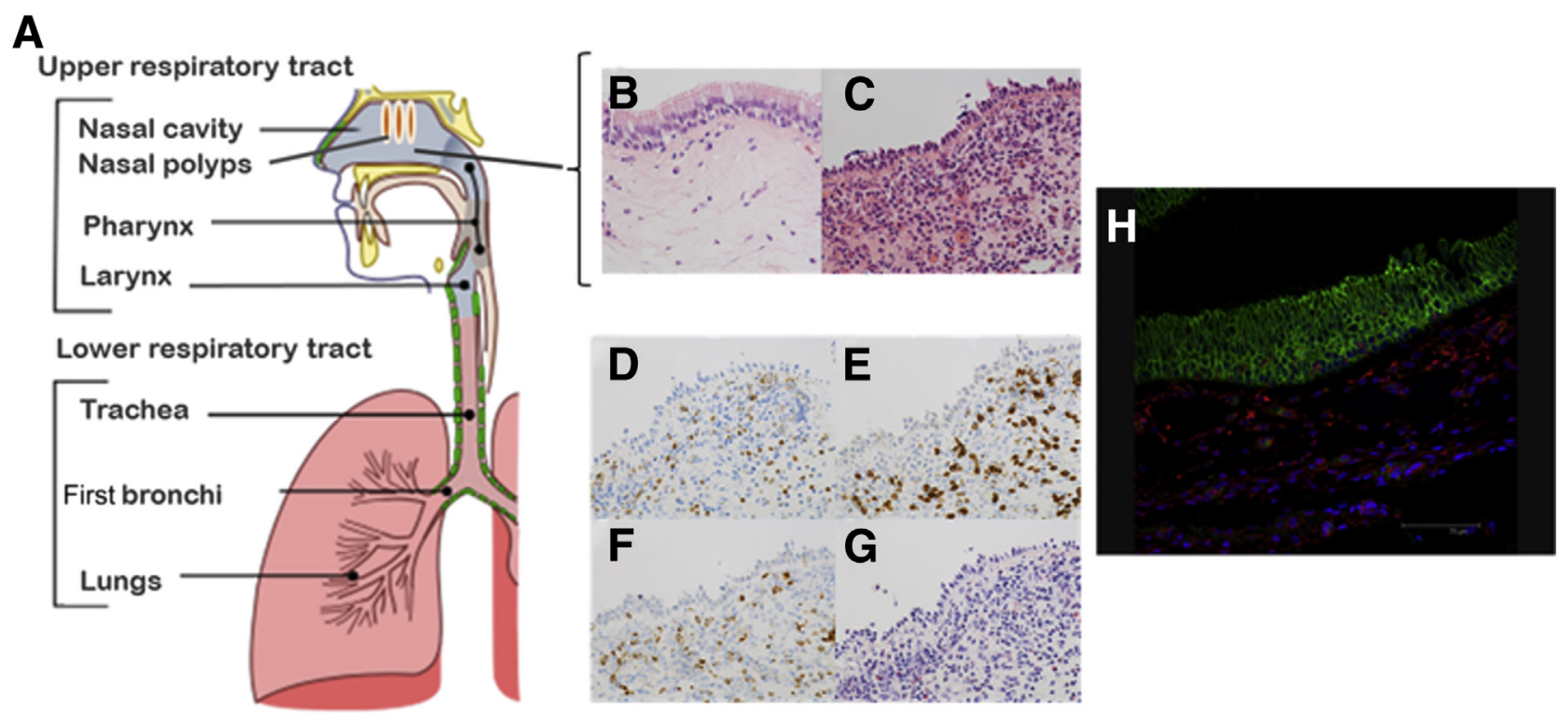

Figure 1 A: Schematic figure of the upper and lower airway anatomy with histologic images of health and upper airway inflammation. Nasal polyps typically originate from the mucosa superiorly in the sinonasal cavities. B-H: Hematoxylin and eosin-stained tissue sections from the upper airway. B: Healthy upper airway mucosa with pseudostratified ciliated respiratory epithelium that lines the sinonasal cavities. C: Typical pathologic findings in chronic rhinosinusitis; epithelial cell loss, infiltration of immune cells in the fibroblast-rich lamina propria, and basement membrane thickening. D-F: Immunohistochemical staining for neutrophils (D), T cells (E), macrophages (F), and eosinophils (G). H: Immunohistochemical staining for epithelial cell marker E-cadherin-fluorescein isothiocyanate (green), fibroblast marker vimentin-tetramethylrhodamine (red), and DAPI nuclear stain (blue) to aid localization of inflammatory cells. Histology was ethically approved by the National Research Ethics Committee North East (13/NE/0099). Original magnification: $\times 40$ (B-H). 
provide access for topical medical therapy. Functional endoscopic sinus surgery is a widely accepted treatment for CRS, although as with any surgical procedure it is not without complications, including bleeding, ocular complications, or a leakage of cerebrospinal fluid. Functional endoscopic sinus surgery is a time-consuming and expensive intervention, and the high disease recurrence may require frequent re-operation.

Resection of diseased surgical specimens in functional endoscopic sinus surgery procedures provides a valuable source of tissue for investigation. Histologic analysis of CRS specimens has identified inflammatory cells, inflammatory cytokine signatures, and airway remodeling, although the precise pathophysiological mechanisms that cause the persistent exaggerated sinonasal inflammation remain unclear. This lack of disease understanding perhaps may explain the high proportion of patients refractory to current conventional pharmacologic therapy. The position of the sinonasal tract makes it highly accessible to the delivery of topical medical therapy. As a result, there are tremendous translational research opportunities to develop better topical nasal anti-inflammatory approaches to treat this large patient population and hence reduce the number of surgical procedures.

\section{Disease Pathology and Classification}

The current literature defines CRS as a disease of the nasal and paranasal sinus mucosa present for $>12$ weeks with mucosal changes that vary from inflammatory remodeling to the formation of nasal polyps. ${ }^{10}$ It represents a spectrum of diseases with a common end result of chronic sinonasal inflammation and fibrotic airway remodeling. CRS is subtyped principally by the presence or absence of nasal polyps on examination of the nose either by direct inspection, endoscopic assessment, or computerized tomography imaging. The etiopathogenesis of CRS, however, is poorly understood. It is classified as sinonasal inflammation, but currently is defined only by symptomatology rather than a specific cellular or histologic appearance. The symptoms often are attributable to changes in the sinonasal mucosa, mucus, or mucociliary clearance, leading to sinonasal ostial blockage and impaired function.

Histologic assessment of polypoid and nonpolypoid tissue specimens shows that they represent differing disease pathologies. CRS without nasal polyps is characterized by sinonasal fibrosis, basement membrane thickening, epithelial damage, mononuclear cell infiltration, and goblet cell hyperplasia. ${ }^{12}$ CRS with nasal polyps is characterized by basement membrane thickening, epithelial damage, stromal edema, and pseudocyst formation. ${ }^{12,13}$ The basement membrane thickening is the end result of a dense fibrotic response typified by accumulation of fibronectin and type I, III, and V collagens. ${ }^{14}$ Both diseases also have a differing Thelper cell profile and cytokine signature. CRS without nasal polyps has been shown to be predominantly a Th1 inflammatory environment with type 1 interferon (IFN)- $\gamma$ as the dominant cytokine along with the profibrotic transforming growth factor (TGF)- $\beta$. In contrast, CRS with nasal polyps is associated with a Th2 and interleukin (IL)-5 dominant inflammatory environment. ${ }^{15}$ The pathophysiology of the profibrotic and proinflammatory processes in CRS currently is being investigated by concerted international efforts.

\section{Cellular Sources of Inflammation in the Upper Airway}

Sinonasal chronic inflammation is not typically seen simultaneously in other organs of CRS sufferers. Chronic inflammatory conditions have two defining features: chronicity and tissue specificity; for example, dermatitis, arthritis, colitis, and so forth. What determines the principal site of occurrence? It would seem likely that dysregulation of inflammatory pathways is local rather than systemic, otherwise the body would be consumed by inflammation. Recent research has highlighted the role of stromal cells in the generation and persistence of chronic inflammation. ${ }^{3}$ Rather than simply being scaffolding or extracellular matrix-secreting cells on which organ systems are built, stromal cells including fibroblasts and osteocytes have their own independent immunologic functions. Moreover, stromal cells from different tissues are significantly different, for example, fibroblasts from inflamed skin have a completely different appearance, immunologic profile, and function from fibroblasts from arthritic joints. These potential epigenetic changes are stable over generations, both in vivo and in vitro. ${ }^{16}$ It has been suggested that stromal cells may orchestrate tissue-specific chronic inflammation, directing and activating immune cells. ${ }^{17}$ Such a hypothesis could be assigned readily to the paranasal sinuses, where epithelial cells reside on a dense network of fibroblasts within a complex bony honeycomb. Recent studies in early CRS have suggested that an increase in sinonasal fibroblasts and collagen deposition precedes local inflammation, ${ }^{18}$ not vice versa. The initiator of fibroblast recruitment and expansion is suggested to be owing to the up-regulation of TGF- $\beta$, a key cytokine in wound healing and repair. This novel concept opposes the traditional concept that fibrosis and airway remodeling result from epithelial injury.

Examination of sinonasal tissue sections from CRS patients and healthy controls shows a marked difference in their tissue architecture. CRS patients undergo airway remodeling with epithelial damage and an influx of multiple immune cell types including neutrophils, eosinophils, $\mathrm{T}$ cells, and macrophages. This inflammatory infiltrate is localized alongside fibroblasts in the lamina propria underneath the epithelial surface. At present, there are no therapeutic approaches that target the fibroblast in upper airway pathology. Here, we review our current understanding of the 


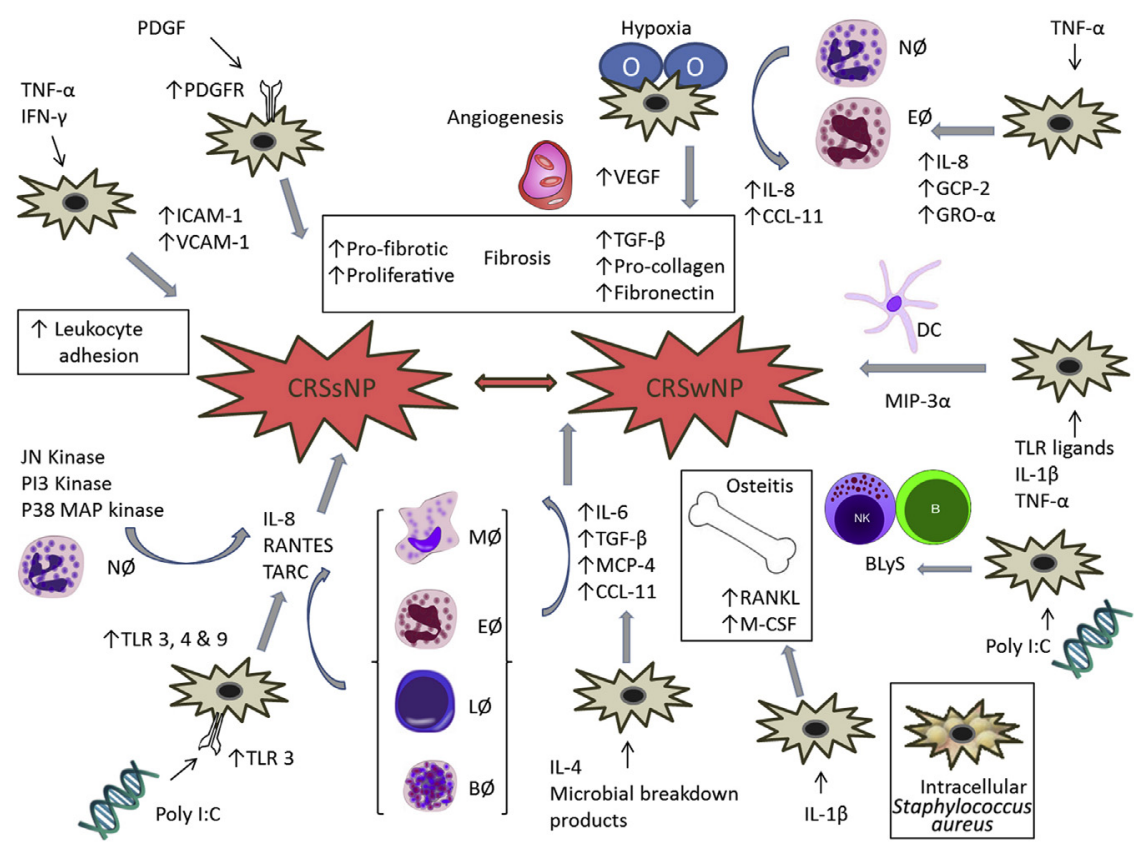

$\underbrace{2}_{2}$ Fibroblast
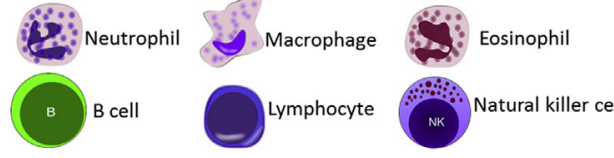

Figure 2 Summary of the current inflammatory and fibrotic roles of the fibroblast in chronic rhinosinusitis with nasal polyposis (CRSwNP) and without nasal polyposis (CRSsNP). Sinonasal fibroblasts have been shown to have a wide range of chemotactic, inflammatory, and profibrotic roles in the pathophysiology of chronic rhinosinusitis. BLyS, B-lymphocyte stimulator; CCL-11, C-C motif chemokine-11; DC, dendritic cell; GCP-2, granulocyte chemotactic protein-2; GRO- $\alpha$, growthrelated oncogene $\alpha$; ICAM, intercellular adhesion molecule; IFN, interferon; MCP-4, monocyte chemotactic protein-4; MIP-3 $\alpha$, macrophage inflammatory protein- $3 \alpha$; NK, natural killer; PDGF, platelet-derived growth factor; PDGFR, plateletderived growth factor receptor; PI, phosphatidylinositol; TGF, transforming growth factor; TLR, Tolllike receptor; TNF, tumor necrosis factor; VEGF, vascular endothelial growth factor. role of the fibroblast in sinonasal disease with and without nasal polyposis (Figure 2) and how it could be a focus for the development of future CRS-specific therapeutics.

\section{Chronic Rhinosinusitis with Nasal Polyps}

Fibroblasts contribute to the development of nasal polyps, providing extracellular matrix proteins including collagens, fibronectin, and vimentin to the nasal polyp architecture. In addition to this profibrotic role, fibroblasts also have been postulated to have inflammatory actions that may be important in the etiology and persistence of nasal polyps. The majority of investigations to date have been based around in vitro cellular cultures of primary human nasal fibroblasts because animal models of sinonasal disease do not presently sufficiently resemble CRS. ${ }^{19}$

\section{Hypoxia-Driven Inflammation}

Sinonasal mucosal inflammation and swelling as seen in CRS with nasal polyps cause ostial sinus blockage, greatly reducing the sinuses' capacity to ventilate normally. Such processes may generate an environment with reduced oxygen tension. In hepatic fibrosis and many similar conditions, hypoxia results in an infiltration of inflammatory cells and subsequent cytokine release. Early et $\mathrm{al}^{20}$ investigated the role of hypoxia and anoxia on nasal polyp-derived fibroblasts and discovered that nasal polyp fibroblast hypoxia results in an increase in vascular endothelial growth factor,
TGF- $\beta$, IL-8, and C-C motif chemokine-11, which is involved in eosinophil recruitment. Hypoxia also resulted in airway remodeling with significant up-regulation of fibroblast-derived procollagen and fibronectin within the cells. Shun et $\mathrm{al}^{21}$ and Sun et $\mathrm{al}^{22}$ showed similar findings in agreement with nasal polyp fibroblast hypoxia stimulating IL-8 and vascular endothelial growth factor, suggesting they prime the sinonasal environment for neutrophil infiltration and angiogenesis.

\section{Fibroblast-0steitis Crosstalk}

Inflammation and remodeling of the bony paranasal sinus cavities or osteitis has been observed as a factor in CRS pathophysiology. In CRS the histologic and radiologic appearances of the ethmoid sinus bone shows fibrosis, new bone formation, inflammatory infiltrates, and increased bone turnover, similar to skeletal osteomyelitis. ${ }^{23}$ Park et $\mathrm{al}^{24}$ investigated whether CRS inflammation stimulates nasal fibroblasts to function in a manner similar to osteoblasts and disrupt the normal paranasal sinus bone homeostasis. They found that the proinflammatory cytokine IL-1 $\beta$ stimulated nasal polyp fibroblasts to express receptor activator of NF$\kappa \mathrm{B}$ ligand and macrophage colony-stimulating factor, key regulators of osteoclastogenesis. Paranasal fibroblasts reside in a densely packed lamina propria directly in contact with paranasal sinus bone and periosteum. Through this anatomic relationship it would appear that the fibroblast can influence the environment of the paranasal sinus bony labyrinth. 
Whether or not inflammation and osteitis of the bony architecture of the sinuses acts as a reservoir to maintain persistence of the chronic inflammation is currently a subject of much debate. ${ }^{25-27}$

\section{Nasal Polyp Fibroblasts as a Source of Cytokines}

Nasal polyposis is characterized by a chronic Th2 cytokinedominant environment. Evidence is emerging that nasal and airway fibroblasts may be a major source of Th2 cytokines. ${ }^{28-30}$ Nonaka et $\mathrm{al}^{31}$ investigated whether either the Th2 cytokine IL-4 or microbial breakdown products stimulated nasal polyp fibroblasts to produce the C-C chemokine monocyte chemotactic protein-4. Monocyte chemotactic protein-4 is a potent chemokine for eosinophils, monocytes, and lymphocytes that are all important immune cells in the nasal polyp. ${ }^{32,33}$ Nonaka et $\mathrm{al}^{31}$ assessed the contribution of IL-4 to fibroblast-mediated inflammation in nasal polyposis first by evaluating the presence of IL-4 and proinflammatory lipid receptors on nasal polyp fibroblasts. They reported that IL-4 receptors are present on nasal fibroblasts and that IL-4-stimulated fibroblasts up-regulated the amounts of IL-6, C-C motif chemokine-11, monocyte chemotactic protein- 4 , and TGF- $\beta 1$. The study by Steinke et $\mathrm{al}^{34}$ suggested that nasal fibroblasts contribute to ongoing inflammatory processes in nasal polyps, producing an environment to drive nasal polyp growth by the release of proinflammatory IL- 6 and profibrotic TGF- $\beta$ that may work in an autocrine fashion.

\section{Nasal Polyp Fibroblast Chemotaxis of Airway Immune Cells}

Neutrophilic infiltrate of nasal mucosa in CRS is readily identified histologically. The presence of neutrophils suggests that during the development of CRS, neutrophil chemokines are generated. Rudack et $\mathrm{al}^{35}$ isolated nasal polyp fibroblasts and treated them with TNF- $\alpha$. Neutrophil chemokines were measured by enzyme-linked immunosorbent assay and mRNA expression with biological chemotactic activity identified by three-step high-performance liquid chromatography. They identified that IL-8, granulocyte chemotactic protein-2, and growth-related oncogene $\alpha$ were induced by proinflammatory stimuli, with the most significant neutrophil chemotactic activity resulting from IL-8. Growth-related oncogene $\alpha$ contributed to neutrophil chemotaxis and granulocyte chemotactic protein-2 represented a co-stimulatory chemokine from human nasal polyp fibroblasts. The secretion of IL-8 from CRS sinonasal fibroblasts also suggests that once neutrophils have been attracted, they will produce further IL-8 themselves and amplify the inflammatory process. In turn, neutrophils will release additional IL-1 and IL-6, IFN- $\gamma$, and TNF- $\alpha$ to contribute to the chemotaxis and activation of additional immune cells.
Dendritic cells are critical mediators of antigen surveillance and presentation and can help to amplify adaptive immune responses. They are present in upper airway diseases including CRS with nasal polyps, although their regulation in CRS is not yet clear. Nonaka et $\mathrm{al}^{36}$ investigated the role of macrophage inflammatory protein- $3 \alpha$, a known migratory factor for immature dendritic cells in nasal polyp fibroblasts. Nasal polyp fibroblasts cultured with Tolllike receptor (TLR) 2, 3, 4, 5 ligands, IL-1 $\beta$, and TNF- $\alpha$ induced macrophage inflammatory protein- $3 \alpha$ expression, as quantified by mRNA on real-time RT- and enzymelinked immunosorbent assay measurement of protein levels. They discuss the fact that fibroblasts make up $47 \%$ of the cells present in nasal polyps, ${ }^{37}$ and that because the proportion of activated fibroblasts is significantly higher in nasal polyps than in healthy nasal mucosa, they may well be a critical source of inflammatory mediators. The researchers further proposed that nasal polyp fibroblasts may contribute to dendritic cell recruitment by TLR- and proinflammatory cytokine-induced production of macrophage inflammatory protein-3 $\alpha$.

\section{Nasal Polyp Fibroblast Interactions with the Adaptive Immune System}

Recruitment and activation of B cells to sites of upper airway inflammation will engage the humoral immunity of the adaptive immune system. B-lymphocyte stimulator (BLyS) has potent stimulatory activity on B cells and their subsequent immune responses. Yamada et $\mathrm{al}^{38}$ examined BLyS expression in human nasal polyp fibroblasts. They identified that BLyS was present in nasal polyp fibroblasts and its expression was greatly induced by the viral TLR analog poly(I:C) in a dose-dependent manner. BLyS is an important survival factor for lymphocytes, increasing B-cell, CD4-positive T-cell, and natural killer cell activity. ${ }^{39}$ BLyS is targeted therapeutically in patients with systemic lupus erythematosus with the monoclonal antibody belimumab. The finding that BLyS is overexpressed in nasal polyp tissue may allow future therapeutic trials of similar agents in nasal polyposis.

\section{Intracellular Nasal Polyp Fibroblast Staphylococcus aureus}

Staphylococcus aureus is one of the major pathogens in CRS with nasal polyps and its persistence has been hypothesized as a factor in the chronicity of nasal polyp inflammation. The potential for $S$. aureus to reside intracellularly, thus being protected from extracellular host defense mechanisms, is likely to facilitate its persistence in the nasal environment. It has been established that fibronectin binding proteins on the fibroblast surface facilitate intracellular human $S$. aureus invasion via its associated receptor integrin $\alpha_{5} \beta_{1}{ }^{40}$ After internalization, $S$. aureus is capable of inducing proinflammatory cytokines to exacerbate the 
disease microenvironment. Clement et $\mathrm{al}^{41}$ identified invasion of S. aureus into fibroblast and myofibroblastic cells of CRS patients' nasal mucosa by confocal scanning microscopy. Clusters of $>10$ intracellular $S$. aureus organisms frequently were seen intracellularly encapsulated within a ring of $\alpha$-smooth muscle actin.

In summary, the fibroblast in chronic sinusitis with nasal polyposis appears to have multiple roles, especially in regard to airway remodeling, immune cell chemotaxis, and contributing directly to the inflammatory milieu, which are all hallmarks of nasal polyp formation.

\section{Chronic Rhinosinusitis without Nasal Polyps}

Understandably, because of the plentiful supply of excised nasal polyp tissue, the majority of sinonasal fibroblast investigations have been performed using fibroblasts derived from patients with nasal polyps. However, nasal fibroblasts are readily isolated from nonpolypoid tissue, a feature that has been exploited successfully in a variety of investigations. The clear distinction between the clinical phenotypes of polypoid and nonpolypoid CRS has not yet been mirrored by distinct cellular differences in phenotype and function of their respective CRS fibroblasts. This is no doubt owing to the novelty of the fibroblast-driven upper airway inflammation hypothesis, however, we do know fibroblasts have a number of roles in both CRS with and without polyps. In the sections to follow, we review what is known about fibroblast involvement in nonpolypoid CRS.

\section{Cell Receptors and Inflammatory Signaling in Nasal Fibroblasts}

The sinonasal mucosa is exposed to a large range of pathogens, especially respiratory viruses. Fibroblasts compose a dense submucosal layer of the sinonasal tract and because of their location no doubt convey an important protective role from many common viral infections. Takahashi et $\mathrm{al}^{42}$ investigated the effects of a synthetic double-stranded RNA viral analog, poly(I:C), on chemokines, type I interferons, Th1 cytokines, and proinflammatory cytokines in nasal mucosal fibroblasts. They first confirmed the presence of TLRs on nasal fibroblasts, identifying high levels of TLR 3,4 , and 9. TLRs $1,2,5$, and 6 also were detected but only at low levels, whereas TLR 7, 8, and 10 were absent. poly(I:C) signals via TLR-3 and expression of this receptor increased fivefold after poly(I:C) treatment. A significant release of the chemokines IL- 8 and regulated on activation normal T-cell expressed and secreted (RANTES) and small amounts of type I interferon IFN- $\beta$ also were observed after poly(I:C) treatment. RANTES is a potent chemoattractant for a number of immune cells including monocytes, eosinophils, lymphocytes, and basophils. In the sinonasal environment it has been shown that fibroblasts, not epithelial cells, are the source of RANTES. ${ }^{43}$ Release of eotaxin,
IL- $1 \beta$, TNF- $\alpha$, IFN- $\alpha$, IFN- $\gamma$, and IL-12 was assessed by enzyme-linked immunosorbent assay, but could not be detected after nasal fibroblast stimulation. Takahashi et $\mathrm{al}^{42}$ also confirmed that c-Jun N-terminal kinase and phosphatidylinositol 3 kinase signaling pathways mediated the production of nasal fibroblast-derived IL-8 and RANTES. In addition, p38 mitogen-activated protein kinase was found to be important for IL-8 production.

Therefore, Takahashi et $\mathrm{al}^{42}$ proposed poly(I:C), similar to viruses clinically, are potent and selective stimuli for nasal fibroblast-derived IL-8 and RANTES, but not Th1 cytokines, proinflammatory cytokines, or eotaxin. They suggested that a better understanding of sinonasal viral infection-associated chemokines may offer new targets to pharmacologically block cellular inflammation in the nasal and sinus cavities.

\section{Nasal Fibroblast-Derived Cytokines and Chemokines}

Kouzaki et $\mathrm{al}^{44}$ investigated the role in CRS of plateletderived growth factor, a proliferative and profibrotic cytokine with emerging roles in renal, hepatic, respiratory, and other inflammatory and fibrotic organ pathologies. By using immunohistochemical techniques they identified that platelet-derived growth factor in CRS patients was localized in inflammatory, epithelial, glandular, and vascular endothelial cells. Increased expression of platelet-derived growth factor receptors was found in CRS submucosal fibroblasts. The investigators suggested that in CRS, local plateletderived growth factor production may be important in promoting sinonasal fibrosis.

Nonaka et $\mathrm{al}^{30}$ analyzed the ability of nasal fibroblasts together with fibroblasts from the respiratory tract to contribute to inflammatory cell infiltration via chemokine production. They measured the ability of fibroblasts to produce thymus and activation-regulated chemokine, a Th2 chemokine. They reported that co-stimulation with either TNF- $\alpha$ and poly(I:C) or Th2 cytokines induced a substantial thymus and activation-regulated chemokine release. Nasal fibroblasts therefore may be an additional source of chemokines, amplifying viral and Th2-induced airway disease.

Oyer et $\mathrm{al}^{45}$ studied the role of leukocyte adhesion molecules vascular cell adhesion molecule (VCAM) and intercellular adhesion molecule (ICAM) to attract neutrophils and eosinophils by nasal fibroblasts. Levels of nasal fibroblast VCAM and ICAM were measured by flow cytometry. They found that both ICAM and VCAM nasal fibroblast expression was increased in CRS. In addition, in vitro treatment with TNF- $\alpha$ and IFN- $\gamma$ further increased ICAM, and treatment with TNF- $\alpha$ and IL-4 increased VCAM. From these observations they suggested that CRS has higher levels of leukocyte adhesion molecules, and the effect was amplified by the CRS inflammatory cytokine environment.

Taken together, it would suggest that the fibroblasts in chronic rhinosinusitis without nasal polyps are important 
sensors of the sinonasal environment, able to monitor and respond to the upper airway environment through expression of a variety of pattern recognition receptors. Dependent on their precise environmental milieu, nasal fibroblasts are able to produce a variety of proinflammatory cytokines and chemokines to amplify the local inflammatory response.

\section{Future Therapeutic Options}

The emerging role of the fibroblast in the pathogenesis of chronic sinonasal disorders opens up novel avenues for pharmacologic treatments. Currently, there are unsurprisingly no clinical trials of therapeutics targeting the fibroblast in sinusitis because fibroblast-driven sinonasal disease remains a novel concept, although this may well change in the near future. To date, we have identified a few published reports that use human nasal polyp fibroblasts in early therapeutic investigations.

Nasal polyp fibroblasts have been used in experiments to investigate the potential anti-inflammatory effect of dehydroxymethylepoxyquinomicin, an NF- $\mathrm{KB}$ inhibitor either alone or in combination with corticosteroids (fluticasone propionate). ${ }^{46}$ Corticosteroids act as negative regulators of transcription through inhibition of transcription factors, especially NF- $\kappa \mathrm{B} .{ }^{47}$ Higher expression of NF- $\kappa \mathrm{B}$ has been reported in chronic rhinosinusitis with nasal polyps, ${ }^{48}$ therefore inhibition would be a logical approach. Nasal polyp fibroblasts were cultured in media enriched with TNF- $\alpha$ in three separate concentrations of steroid, DHMEQ, or both together. The inflammatory response was measured via levels of RANTES, VCAM-1, and ICAM-1 expression by both RT-PCR and enzyme-linked immunosorbent assay. The nuclear translocation of NF- $\kappa \mathrm{B}$ also was evaluated. Both fluticasone propionate and DHMEQ inhibited the fibroblast inflammatory response and NF- $\kappa \mathrm{B}$ nuclear translocation, and their combined effect was found to be synergistic. Given that corticosteroid treatment failure is one of the indications for surgical therapy, DHMEQ may offer promise of a new treatment adjunct to treat nasal polyps.

Vitamin D analogs have emerged recently as a family of antiproliferative agents in addition to their classic role in calcium homeostasis. These antiproliferative properties are being considered for the sinonasal cavity to target nasal polyp growth. Rostkowska-Nadolska et $\mathrm{al}^{49}$ and Fraczek et $\mathrm{al}^{50}$ investigated the effect of two vitamin $\mathrm{D}$ derivatives, calcitriol and tacalcitol, as monotherapy and in combination with a corticosteroid on nasal polyp fibroblast proliferation. Both vitamin D derivatives showed promising fibroblast antiproliferative activity as single agents and in combination with budesonide. Tacalcitol showed the most promising combination of higher fibroblast antiproliferative activity, suppressing RANTES and other chemokines in a dosedependent manner, combined with lower cellular toxicity. Approaches using vitamin D analogs therefore may offer the potential for novel topical therapeutic options in sinonasal diseases.

Our understanding of the potential role of the fibroblast in inflammatory upper airway disorders is emerging, although it is by no means complete. To date, we understand that sinonasal fibroblasts have a variety of functions in addition to traditional extracellular matrix synthesis, the majority of which appear to influence immune cell chemotaxis and amplify the inflammatory response. These findings in the upper airway replicate and further confirm the role of fibroblasts as seen in many other diseases characterized by inflammation and fibrosis. Fibroblasts within the upper airway may not simply be innocent bystanders in the pathophysiology of chronic sinusitis. From these early studies there will no doubt be an expansion in contemporary sinusitis, fibroblast, and inflammatory research to enhance our understanding of these disease mechanisms. The potential for stromal cells to shape and define the inflammatory environment of the sinonasal cavity is an exciting new disease paradigm and offers the much-needed potential for a different treatment approach to some of our most common conditions.

\section{References}

1. Boor P, Ostendorf T, Floege J: Renal fibrosis: novel insights into mechanisms and therapeutic targets. Nat Rev Nephrol 2010, 6: 643-656

2. Mehal WZ, Iredale J, Friedman SL: Scraping fibrosis: expressway to the core of fibrosis. Nat Med 2011, 17:552-553

3. Naylor AJ, Filer A, Buckley CD: The role of stromal cells in the persistence of chronic inflammation. Clin Exp Immunol 2013, 171: $30-35$

4. Barry-Hamilton V, Spangler R, Marshall D, McCauley S, Rodriguez HM, Oyasu M, Mikels A, Vaysberg M, Ghermazien H, Wai C, Garcia CA, Velayo AC, Jorgensen B, Biermann D, Tsai D, Green J, Zaffryar-Eliot S, Holzer A, Ogg S, Thai D, Neufeld G, Van Vlasselaer P, Smith V: Allosteric inhibition of lysyl oxidase-like-2 impedes the development of a pathologic microenvironment. Nat Med 2010, 16:1009-1017

5. Loomis-King H, Flaherty KR, Moore BB: Pathogenesis, current treatments and future directions for idiopathic pulmonary fibrosis. Curr Opin Pharmacol 2013, 13:377-385

6. Meltzer EO, Hamilos DL, Hadley JA, Lanza DC, Marple BF, Nicklas RA, Bachert C, Baraniuk J, Baroody FM, Benninger MS, Brook I, Chowdhury BA, Druce HM, Durham S, Ferguson B, Gwaltney JM, Kaliner M, Kennedy DW, Lund V, Naclerio R, Pawankar R, Piccirillo JF, Rohane P, Simon R, Slavin RG, Togias A, Wald ER, Zinreich SJ; American Academy of Allergy, Asthma and Immunology (AAAAI); American Academy of Otolaryngic Allergy (AAOA); American Academy of Otolaryngology-Head and Neck Surgery (AAO-HNS); American College of Allergy, Asthma and Immunology (ACAAI); American Rhinologic Society: Rhinosinusitis: establishing definitions for clinical research and patient care. J Allergy Clin Immunol 2004, 114:155-212

7. Hastan D, Fokkens WJ, Bachert C, Newson RB, Bislimovska J, Bockelbrink A, Bousquet PJ, Brozek G, Bruno A, Dahlén SE, Forsberg B, Gunnbjörnsdóttir M, Kasper L, Krämer U, Kowalski ML, Lange B, Lundbäck B, Salagean E, Todo-Bom A, Tomassen P, Toskala E, van Drunen CM, Bousquet J, Zuberbier T, 
Jarvis D, Burney P: Chronic rhinosinusitis in Europe-an underestimated disease. A GA(2)LEN study. Allergy 2011, 66: $1216-1223$

8. Ray NF, Baraniuk JM, Thamer M, Rinehart CS, Gergen PJ, Kaliner M, Josephs S, Pung YH: Healthcare expenditures for sinusitis in 1996: contributions of asthma, rhinitis, and other airway disorders. J Allergy Clin Immunol 1999, 103:408-414

9. Lal D, Scianna JM, Stankiewicz JA: Efficacy of targeted medical therapy in chronic rhinosinusitis, and predictors of failure. Am J Rhinol Allergy 2009, 23:396-400

10. Fokkens WJ, Lund VJ, Mullol J, Bachert C, Alobid I, Baroody F, Cohen N, Cervin A, Douglas R, Gevaert P, Georgalas C, Goossens H, Harvey R, Hellings P, Hopkins C, Jones N, Joos G, Kalogjera L, Kern B, Kowalski M, Price D, Riechelmann H, Schlosser R, Senior B, Thoimas M, Toskala E, Voegels R, Wang de Y, Wormald PJ: European position paper on rhinosinusitis and nasal polyps 2012. Rhinol Suppl 2012:1-298

11. Dubin MG, Liu C, Lin SY, Senior BA: American Rhinologic Society member survey on "maximal medical therapy" for chronic rhinosinusitis. Am J Rhinol 2007, 21:483-488

12. Kou W, Hu GH, Yao HB, Wang XQ, Shen Y, Kang HY, Hong SL: Regulation of transforming growth factor-betal activation and expression in the tissue remodeling involved in chronic rhinosinusitis. ORL J Otorhinolaryngol Relat Spec 2012, 74: $172-178$

13. Bachert C, Gevaert P, Holtappels G, Cuvelier C, van Cauwenberge P: Nasal polyposis: from cytokines to growth. Am J Rhinol 2000, 14: 279-290

14. Pawankar R, Nonaka M: Inflammatory mechanisms and remodeling in chronic rhinosinusitis and nasal polyps. Curr Allergy Asthma Rep 2007, 7:202-208

15. Van Crombruggen K, Zhang N, Gevaert P, Tomassen P, Bachert C: Pathogenesis of chronic rhinosinusitis: inflammation. J Allergy Clin Immunol 2011, 128:728-732

16. Polyak K, Weinberg RA: Transitions between epithelial and mesenchymal states: acquisition of malignant and stem cell traits. Nat Rev Cancer 2009, 9:265-273

17. Suwara MI, Green NJ, Borthwick LA, Mann J, Mayer-Barber KD, Barron L, Corris PA, Farrow SN, Wynn TA, Fisher AJ, Mann DA: IL-1alpha released from damaged epithelial cells is sufficient and essential to trigger inflammatory responses in human lung fibroblasts. Mucosal Immunol 2014, 7:684-693

18. Van Bruaene N, C PN, Van Crombruggen K, De Ruyck N, Holtappels G, Van Cauwenberge P, Gevaert P, Bachert C: Inflammation and remodelling patterns in early stage chronic rhinosinusitis. Clin Exp Allergy 2012, 42:883-890

19. Kara CO: Animal models of sinusitis: relevance to human disease. Curr Allergy Asthma Rep 2004, 4:496-499

20. Early SB, Hise K, Han JK, Borish L, Steinke JW: Hypoxia stimulates inflammatory and fibrotic responses from nasal-polyp derived fibroblasts. Laryngoscope 2007, 117:511-515

21. Shun CT, Lin SK, Hong CY, Huang HM, Liu CM: Hypoxia induces cysteine-rich 61, vascular endothelial growth factor, and interleukin-8 expressions in human nasal polyp fibroblasts: an implication of neutrophils in the pathogenesis of nasal polyposis. Am J Rhinol Allergy 2011, 25:15-18

22. Sun D, Matsune S, Ohori J, Fukuiwa T, Ushikai M, Kurono Y: TNFalpha and endotoxin increase hypoxia-induced VEGF production by cultured human nasal fibroblasts in synergistic fashion. Auris Nasus Larynx 2005, 32:243-249

23. Kennedy DW, Senior BA, Gannon FH, Montone KT, Hwang P, Lanza DC: Histology and histomorphometry of ethmoid bone in chronic rhinosinusitis. Laryngoscope 1998, 108:502-507

24. Park CS, Park YS, Park YJ, Cho JH, Kang JM, Kim SY: The inhibitory effects of macrolide antibiotics on bone remodeling in chronic rhinosinusitis. Otolaryngol Head Neck Surg 2007, 137: 274-279
25. Georgalas C, Videler W, Freling N, Fokkens W: Global Osteitis Scoring Scale and chronic rhinosinusitis: a marker of revision surgery. Clin Otolaryngol 2010, 35:455-461

26. Sacks PL, Snidvongs K, Rom D, Earls P, Sacks R, Harvey RJ: The impact of neo-osteogenesis on disease control in chronic rhinosinusitis after primary surgery. Int Forum Allergy Rhinol 2013, 3: 823-827

27. Videler WJ, Georgalas C, Menger DJ, Freling NJ, van Drunen CM, Fokkens WJ: Osteitic bone in recalcitrant chronic rhinosinusitis. Rhinology 2011, 49:139-147

28. Tremblay GM, Jordana M, Gauldie J, Sarnstrand B: Fibroblasts as effector cells in fibrosis. New York, NY, Marcel Dekker, 1995, pp $541-577$

29. Nonaka M, Pawankar R, Saji F, Yagi T: Distinct expression of RANTES and GM-CSF by lipopolysaccharide in human nasal fibroblasts but not in other airway fibroblasts. Int Arch Allergy Immunol 1999, 119:314-321

30. Nonaka M, Ogihara N, Fukumoto A, Sakanushi A, Kusama K, Pawankar R, Yagi T: Combined stimulation with Poly(I:C), TNFalpha and Th2 cytokines induces TARC production by human fibroblasts from the nose, bronchioles and lungs. Int Arch Allergy Immunol 2010, 152:327-341

31. Nonaka M, Fukumoto A, Ogihara N, Pawankar R, Sakanushi A, Yagi T: Expression of MCP-4 by TLR ligand-stimulated nasal polyp fibroblasts. Acta Otolaryngol 2007, 127:1304-1309

32. Cauna N, Manzetti GW, Hinderer KH, Swanson EW: Fine structure of nasal polyps. Ann Otol Rhinol Laryngol 1972, 81:41-58

33. Nonaka M, Nonaka R, Woolley $\mathrm{K}$, Adelroth E, Miura K, Okhawara Y, Glibetic M, Nakano K, O’Byrne P, Dolovich J, Jordana M: Distinct immunohistochemical localization of IL-4 in human inflamed airway tissues. IL-4 is localized to eosinophils in vivo and is released by peripheral blood eosinophils. J Immunol 1995, 155:3234-3244

34. Steinke JW, Crouse CD, Bradley D, Hise K, Lynch K, Kountakis SE, Borish L: Characterization of interleukin-4stimulated nasal polyp fibroblasts. Am J Respir Cell Mol Biol 2004, 30:212-219

35. Rudack C, Hermann W, Eble J, Schroeder JM: Neutrophil chemokines in cultured nasal fibroblasts. Allergy 2002, 57:1159-1164

36. Nonaka M, Ogihara N, Fukumoto A, Sakanushi A, Kusama K, Pawankar R, Yagi T: Nasal polyp fibroblasts produce MIP-3alpha in response to toll-like receptor ligands and cytokine stimulation. Rhinology 2010, 48:41-46

37. Jordana M, Dolovich J, Ohno I, Finotto S, Denburg J: Nasal polyposis: a model for chronic inflammation. Boston, MA, Blackwell Scientific Publications, 1995, pp 156-164

38. Yamada T, Lizhong S, Takanashi N, Kubo S, Narita N, Suzuki D, Takabayashi T, Kimura Y, Fujieda S: Poly(I:C) induces BLySexpression of airway fibroblasts through phosphatidylinositol 3-kinase. Cytokine 2010, 50:163-169

39. Shan X, Chen L, Cao M, Xu L, Zhang S: Effects of human soluble BAFF synthesized in Escherichia coli on $\mathrm{CD} 4+$ and CD8 + T lymphocytes as well as NK cells in mice. Physiol Res 2006, 55:301-307

40. Alexander EH, Hudson MC: Factors influencing the internalization of Staphylococcus aureus and impacts on the course of infections in humans. Appl Microbiol Biotechnol 2001, 56:361-366

41. Clement S, Vaudaux P, Francois P, Schrenzel J, Huggler E, Kampf S, Chaponnier C, Lew D, Lacroix JS: Evidence of an intracellular reservoir in the nasal mucosa of patients with recurrent Staphylococcus aureus rhinosinusitis. J Infect Dis 2005, 192:1023-1028

42. Takahashi N, Yamada T, Narita N, Fujieda S: Double-stranded RNA induces production of RANTES and IL-8 by human nasal fibroblasts. Clin Immunol 2006, 118:51-58

43. Maune S, Berner I, Sticherling M, Kulke R, Bartels J, Schröder JM: Fibroblasts but not epithelial cells obtained from human nasal 
mucosa produce the chemokine RANTES. Rhinology 1996, 34: $210-214$

44. Kouzaki H, Seno S, Fukui J, Owaki S, Shimizu T: Role of plateletderived growth factor in airway remodeling in rhinosinusitis. Am J Rhinol Allergy 2009, 23:273-280

45. Oyer SL, Nagel W, Mulligan JK: Differential expression of adhesion molecules by sinonasal fibroblasts among control and chronic rhinosinusitis patients. Am J Rhinol Allergy 2013, 27:381-386

46. Valera FC, Umezawa K, Brassesco MS, Castro-Gamero AM, Queiroz RG, Scrideli CA, Tone LG, Anselmo-Lima WT: Suppression of inflammatory cytokine secretion by an NF-kB inhibitor DHMEQ in nasal polyps fibroblasts. Cell Physiol Biochem 2012, 30:13-22
47. Adcock IM, Caramori G: Cross-talk between pro-inflammatory transcription factors and glucocorticoids. Immunol Cell Biol 2001, 79:376-384

48. Valera FC, Queiroz R, Scrideli C, Tone LG, Anselmo-Lima WT: Expression of transcription factors NF-kappaB and AP-1 in nasal polyposis. Clin Exp Allergy 2008, 38:579-585

49. Rostkowska-Nadolska B, Fraczek M, Gawron W, Latocha M: Influence of vitamin $\mathrm{D}(3)$ analogues in combination with budesonid $\mathrm{R}$ on proliferation of nasal polyp fibroblasts. Acta Biochim Pol 2009, 56:235-242

50. Fraczek M, Rostkowska-Nadolska B, Kusmierz D, Zielinska A, Rok J, Sliupkas-Dyrda E, Grazanka A, Krecicki T, Latocha M: Vitamin D analogs decrease in vitro secretion of RANTES and enhance the effect of budesonide. Adv Med Sci 2012, 57:290-295 